\title{
A Pull-in Operation Mode Accelerometer
}

\author{
Lukasz Pakula, Patrick French \\ Delft University of Technology \\ Micro-electronics and Computer Engineering \\ Delft, The Netherlands \\ 1.pakula@ewi.tudelft.nl
}

\begin{abstract}
In this paper an accelerometer, which measures the pull-in time to obtain the acceleration is presented. As the pullin time is a semi digital signal, the output of the device can be measured with a full digital circuit. No analogue readout circuit is required. The sensitivity and nonlinearity are comparable with the differential capacitive sensing devices. The accelerometer has been fabricated by surface micromachining using $\mathrm{Al}$ as the mechanical layer and phosphosilicate glass as the sacrificial layer. The initial measurements have been performed.
\end{abstract}

\section{INTRODUCTION}

The surface micromachined accelerometers usually have low pull-in voltages and therefore the capacitive sensing is difficult to achieve [1]. W. C. Tang proposed a novel digital accelerometer in 1994 [2], in which the pull-in voltage is measured to obtain the acceleration since the pull-in voltage is the function of the acceleration.

It has been found by the authors that the pull-in time is also a function of the acceleration [3]. An accelerometer, which measures the pull-in time, has been proposed.

The principle of the device is shown in Fig. 1. By applying the pulse voltages $\phi_{1}$ and $\phi_{2}$ to the electrodes 1 and 2 alternately, the mass is pull-in at the stoppers 1 and 2 alternately. $T_{l}$ is the pull-in time from the stopper 1 to 2 , and $T_{2}$ is pull-in tome from the stopper 2 to 1 . When there is no acceleration in the $x$ direction, $T_{1}=T_{2}=T_{0}$. If there is an acceleration in the $x$ direction, the differential pull-in time, $\Delta T=T_{2}-T_{1}$, is proportional to the acceleration. $\Delta T$ is a pulsewidth-modulated signal and can be measured with a digital circuit. The sensitivity and the non-linearity are similar to that of the differential capacitive sensing.

The device has been fabricated by the surface micromachining technology. The pull-in time can be obtained either by measuring the contact between the mass and the stopper or by the capacitive sensing.

\section{PRINCIPLE}

The movement of the mass between the stoppers can be described by the equation:

$$
m \ddot{x}+c \dot{x}+k x=\frac{\varepsilon \varepsilon_{0} A V^{2}}{2\left(d_{0}-x\right)^{2}}+m a
$$

where $a$ is the acceleration, $V$ the driving voltage, $A$ the area of the electrode, $d_{0}$ the initial electrode gap and $x$ the displacement. To keep the device working in the pull-in mode, the driving voltage must be higher than the minimum pull-in voltage and the previous pulse voltage should be held long enough to make sure that the initial condition is $\dot{x}=0$.

To discuss the characteristics in general, a dimensionless equation is preferred. The corresponding dimensionless equation of (1) is

$$
\ddot{\tilde{x}}+2 \dot{\tilde{x}}+\tilde{x}=\frac{\tilde{F}}{(1-\tilde{x})^{2}}+\tilde{a}
$$

where $\tilde{x}=x / d_{0}, \dot{\tilde{x}}=d \tilde{x} / d \tau, \quad \ddot{\tilde{x}}=d^{2} \tilde{x} / d \tau^{2}, \tau=\omega_{0} t$,
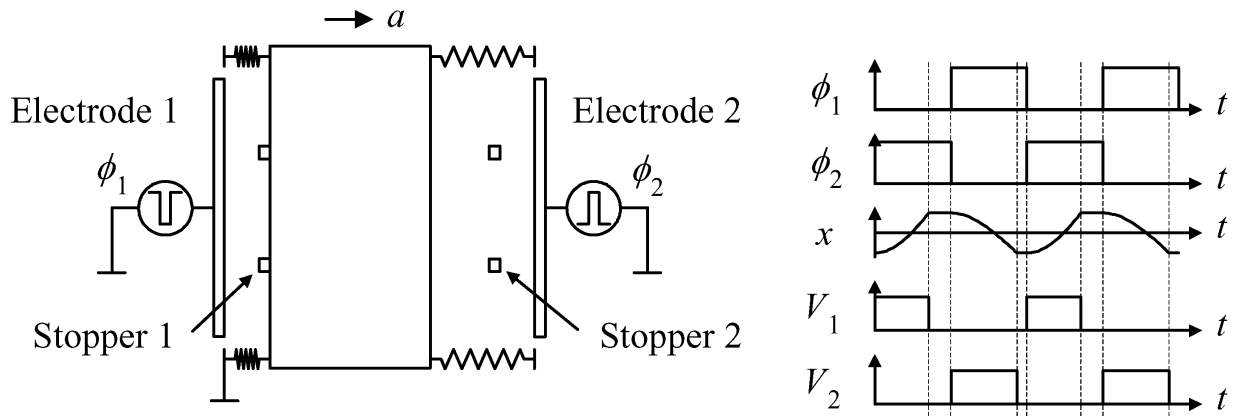

Figure 1. The principle of the pull-in operation mode 
$\omega_{0}$ the circular resonant frequency, $\zeta$ the damping ratio, $\tilde{F}=\varepsilon \varepsilon_{0} A V^{2} /\left(2 k d_{0}^{3}\right)$ the dimensionless driving force and $\tilde{a}=m a / k d_{0}$ the dimensionless acceleration. The driving force must fulfil $\widetilde{F}>4 / 27$, which is corresponding to the minimum pull-in voltage. $\tilde{a}$ is always smaller than 1 according to the definition.

When the damping is zero, the analytical pull-in time can be obtained [3]. The differential pull-in time $\Delta \tau=\tau_{2}-\tau_{1}$ is

$$
\Delta \tau=2 s \tilde{a}+\frac{17}{4} n \tilde{a}^{3}+o\left(\tilde{a}^{3}\right)
$$

where

$$
\begin{aligned}
& s=\int_{-\lambda}^{\lambda}(\lambda+\tilde{x})^{-\frac{1}{2}}\left((\lambda-\tilde{x})+\frac{2 \tilde{F}}{(1-\tilde{x})(1+\lambda)}\right)^{-\frac{3}{2}} d \tilde{x} \\
& n=\int_{-\lambda}^{\lambda}(\lambda+\tilde{x})^{-\frac{1}{2}}\left((\lambda-\tilde{x})+\frac{2 \tilde{F}}{(1-\tilde{x})(1+\lambda)}\right)^{-\frac{7}{2}} d \tilde{x}
\end{aligned}
$$

where $\pm \lambda$ is the positions of the stoppers.

It can be obtained from (3) that $\Delta \tau$ is proportional to the acceleration. The gain of the device can be defined as $G=2 s / \tau_{0}$. Fig. 2 shows the gain with respect to $\tilde{F}$ and $\lambda$. By comparison, the gain of the differential capacitive sensing is 2. Therefore, the gain of the pull-in accelerometer is comparable to that of the normal accelerometer with the differential capacitive sensing.

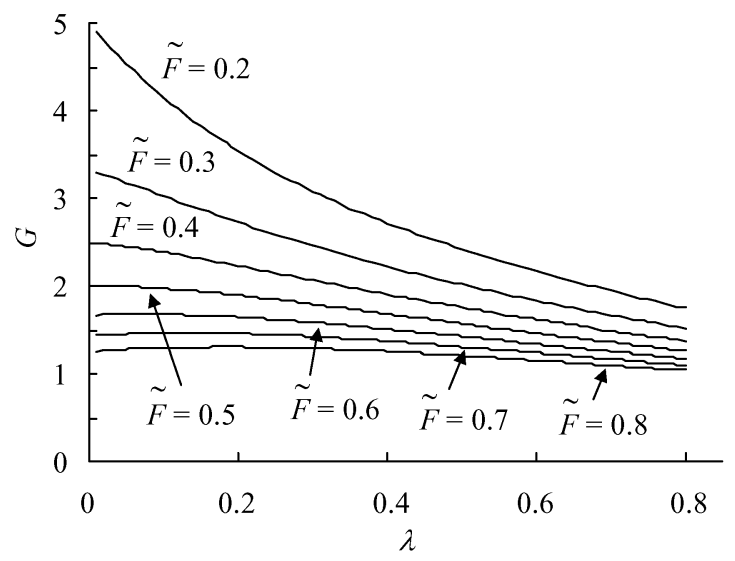

Figure 2. The gain as the function of $\widetilde{F}$ and $\lambda$
The nonlinearity of the pull-in accelerometer is $O\left(\tilde{a}^{3}\right)$, which is the same order of the ratio $(17 / 4) n \tilde{a}^{3} /(2 s \tilde{a})$. The nonlinearity of the pull-in mode is similar to the differential capacitive sensing.

When the damping is non zero but also not high, (2) cannot be solved analytically. Matlab was used to analyse the equation numerically. Fig. 3 shows the gain with respect to the electrostatic force and Fig. 4 the nonlinearity in the range of $\tilde{a} \in[-0.1,0.1]$. $\lambda$ used in the calculations is 0.5 .

It can be observed that both the gain and the nonlinearity increase with the damping and saturate when the damping ratio is larger than 1.

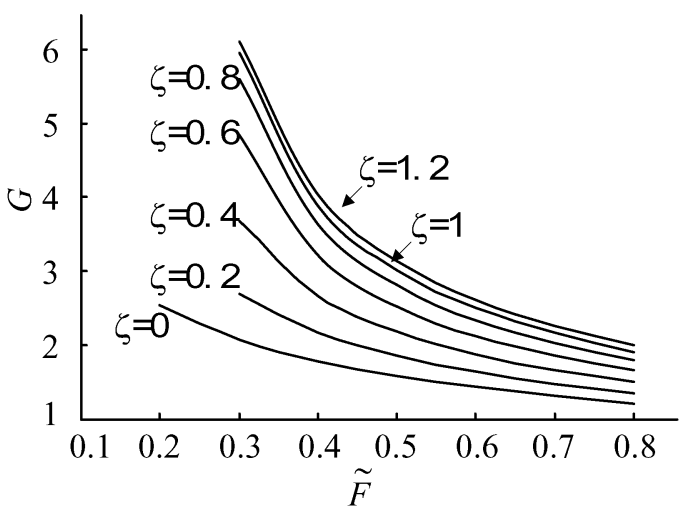

Figure 3. The gain as the function of $\tilde{F}$ and $\zeta . \lambda$ is supposed to be 0.5 .

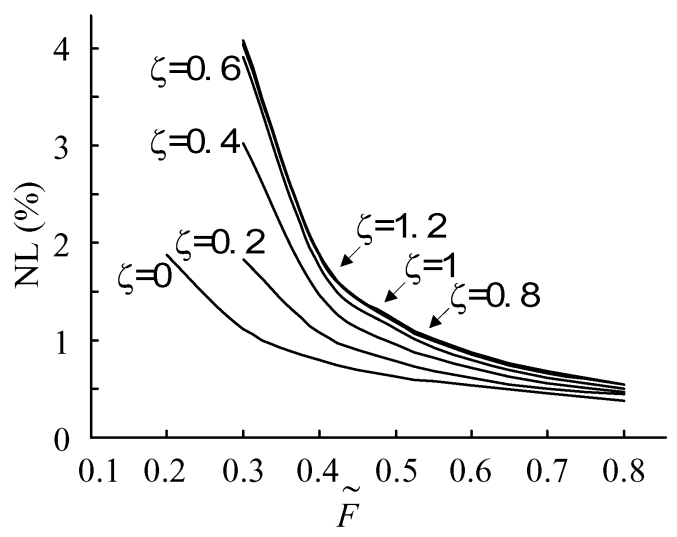

Figure 4. Nonlinearity as the function of $\tilde{F}$ and $\zeta . \lambda$ is supposed to be 0.5 .

As the damping in an accelerometer is usually changed with the displacement, the influence of the variable damping is also analysed. The dominant damping in the system is supposed to be the squeeze film damping, which means that the damping ratio is inversely proportional to the cube of the electrode gap. It is observed that the gain increases with the damping faster than in Fig. 3. 


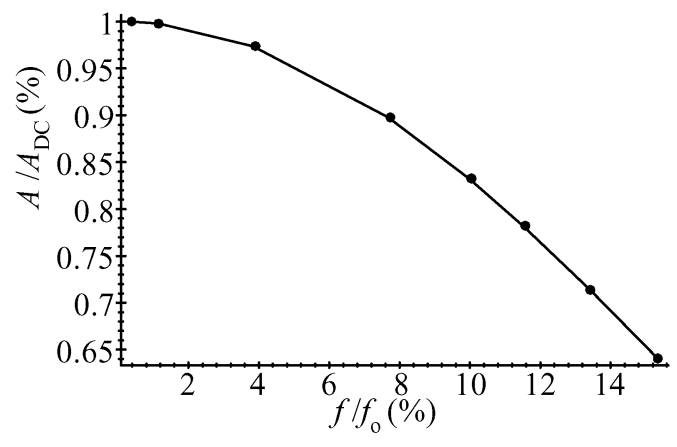

Figure 5. The amplitude-frequency relation

The pull-in operation process is a pulse-width modulation process [4]. The bandwidth of the device is determined by the sampling rate.

In principle, the larger the sampling rate, the larger the bandwidth. The theoretical maximum sampling rate of the pull-in accelerometer is a higher than the resonant frequency. However, the sampling rate is limited by several factors.

Firstly, the sampling pulse width must be larger than the maximum pull-in time, which is determined by the full-scale required.

Secondly, the sampling pulse width must be long enough to make sure that the speed of the mass is 0 before the next pulse because the measurement is sensitive to the initial conditions and the vibration of the mass at the pull-in position causes errors. In this paper, the pulse width of the driving voltage is set to be $1 / f_{0}$, where $f_{0}$ is the resonant frequency. A full measuring circle is $2 / f_{0}$. In other words, the sampling frequency is $f_{\mathrm{o}} / 2$.

Fig. 5 shows the amplitude-frequency relation of a device with $\zeta=0, \lambda=0.5$ and $\widetilde{F}=0.3$. The relation is obtained by Fourier transform of the theoretical output. The bandwidth of the device is $13.4 \%$ of the resonant frequency.

When the acceleration is very large, the structure does not pull-in. The maximum acceleration that can be measured is a function of the operating voltage. To simplify the analysis, the maximum acceleration is estimated with the initial conditions $\tilde{x}=0$ and $\dot{\tilde{x}}=0$.

When the damping is very high, the maximum acceleration is:

$$
|\tilde{a}|<\sqrt[3]{\frac{27 \tilde{F}}{4}}-1
$$

For the device with light damping, the maximum acceleration is larger than (6) due to over-shooting [1]. The maximum acceleration of the device without damping is given by:

$$
|\widetilde{a}|=\sqrt{2 \widetilde{F}}-\frac{1}{2}
$$

\section{DESIGN}

Based on the above analysis the surface micromachined accelerometer has been design. The device structure is made by $3 \mu \mathrm{m}$ thick aluminium. The mass is supported by four folded beams. The mass is $400 \mu \mathrm{m} \times 800 \mu \mathrm{m}$. Each fold of the beam is $2.5 \mu \mathrm{m} \times 150 \mu \mathrm{m}$. Interdigitated electrodes are used to drive the mass. There are 62 fingers on each side of the mass. Each finger is $3 \mu \mathrm{m} \times 100 \mu \mathrm{m}$. The finger-electrode gap is $2 \mu \mathrm{m}$. The mass-stopper gap is $1 \mu \mathrm{m}$. The mass-substrate gap is $1 \mu \mathrm{m}$.

The electrical and mechanical properties have been calculated. The resonant frequency is calculated to be $4 \mathrm{kHz}$. When the displacement is 0 , the damping ratio is calculated to be 0.057 . The pull-in voltage is calculated to be $4.86 \mathrm{~V}$. The pull-in voltage of fingers is higher than $10 \mathrm{~V}$ when the mass is at the pull-in position.

The static and dynamic properties of the accelerometer in pull-in mode are analysed numerically with Matlab software. When the driving voltage is $8 \mathrm{~V}$, the pull-in time is calculated to be $77 \mu$ s. Fig. 6 shows the differential pull-in time with respect to the acceleration, which was obtained by simulation. The non-linearity is $0.87 \% \mathrm{FS}$.

The pull-in time can be obtained either by measuring the contact between the mass and the stopper or by the capacitive sensing.

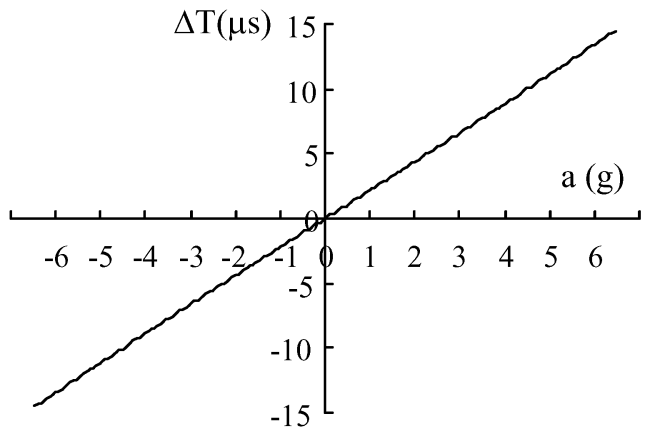

Figure 6. The output under the $8 \mathrm{~V}$ driving voltage

\section{FABRICATION AND MEASUREMENTS}

The device has been fabricated by the surface micromachining technology with $\mathrm{Al}$ as the mechanical layer and PECVD PSG (phosphosilicate glass) as the sacrificial layer, respectively. 
The fabrication process is as follows:

1. First the $1 \mu \mathrm{m}$ PECVD $4 \%$ PSG (4\% of phosphorus in PSG) was deposited on oxidised silicon wafer in Novellus system and patterned to form sacrificial layer.

2. Next the $3 \mu \mathrm{m}$ low temperature Al was sputtered in Trikon Sigma sputter coater. After lithography the layer was patterned in Trikon Omega 201 plasma etcher, forming the device.

3. The structure was released by removing PSG sacrificial layer in $73 \% \mathrm{HF}$ (fluoric acid) with addition of isopropanol. To prevent problem of stiction the freeze drying process was employed after sacrificial step. Fig. 7 shows the SEM photo of the device.

Prior to the packaging the capacitance-voltage measurement was performed. The results are shown in Fig. 8 . The pull-in voltages were measured to be $8.1 \mathrm{~V}$ and $8.7 \mathrm{~V}$ in the positive and the negative directions, respectively. The pull-in voltages are higher than the theoretical values and slightly asymmetric. That could be the explained by the slight deformation of the structure (SEM photo in Fig. 7).

The asymmetry of the pull-in voltages can be compensated by the driving circuit. It can be observed that the resonant frequency changes with the bias voltage. The resonant frequency and the damping ratio at $2 \mathrm{~V} \mathrm{DC}+1 \mathrm{~V}$ $\mathrm{AC}$ are measured to be $4.97 \mathrm{kHz}$ and 0.024 , while the data at $3 \mathrm{~V} \mathrm{DC}+2.18 \mathrm{~V} \mathrm{AC}$ are $4.87 \mathrm{kHz}$ and 0.022 . Initial experiments have shown the change in pull-in time of $5 \mu \mathrm{s}$ from 0 to $1 \mathrm{G}$.
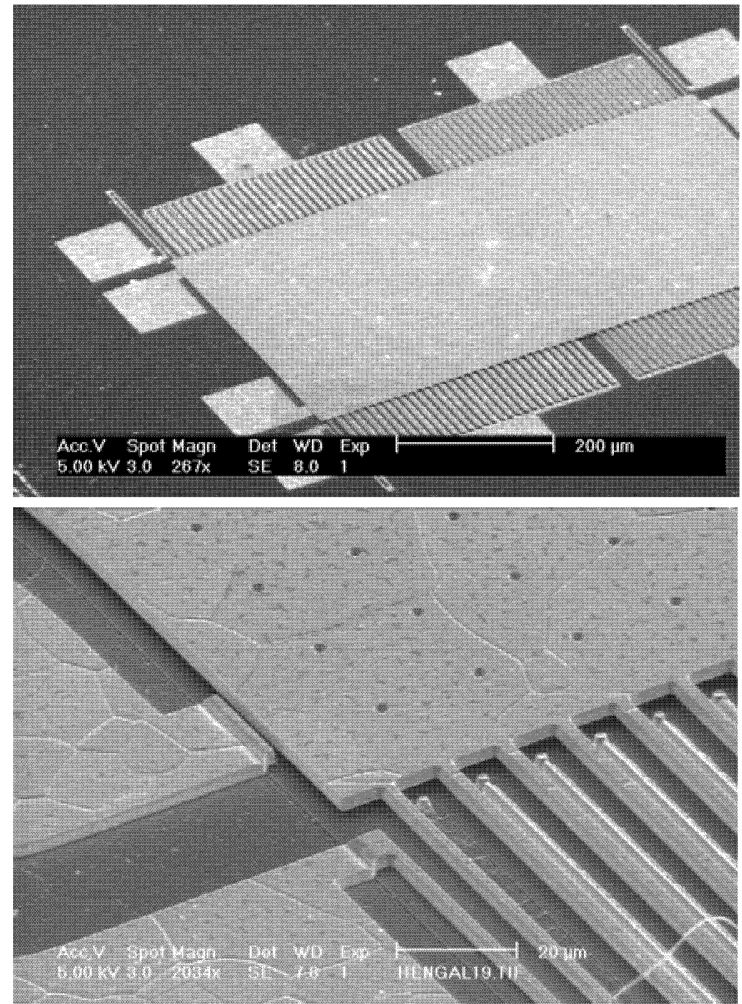

Figure 7. The SEM photo of the device and the close-ups of the stopper and the fingers.

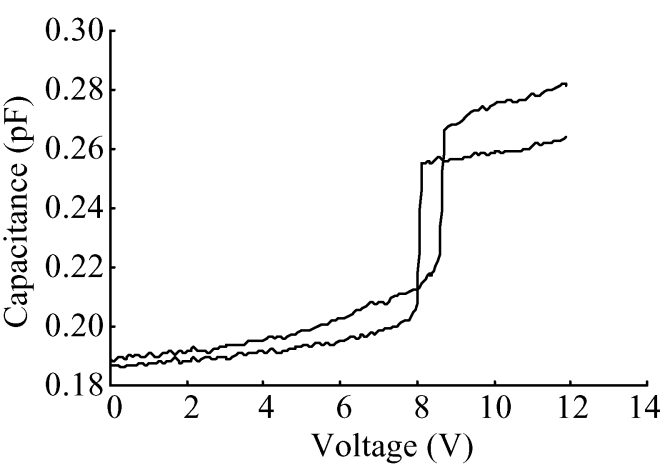

Figure 8. CV measurement

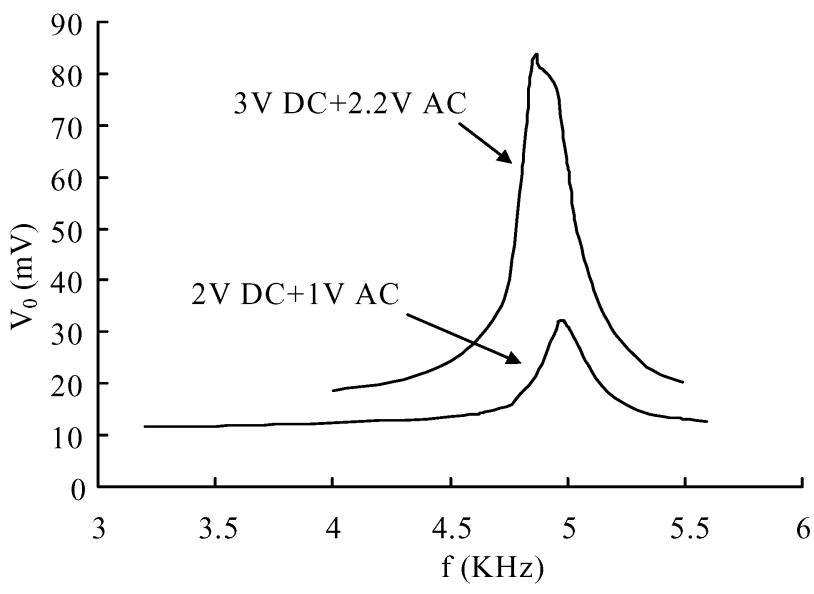

Figure 9. Frequency response

\section{SUMMARY}

A pull-in accelerometer, which features a digital output, has been designed and fabricated by surface micromachining technology. The pull-in voltages have been measured. The pull-in time measured from 0 to $1 \mathrm{G}$ is $5 \mu \mathrm{s}$

\section{ACKNOWLEDGEMENTS}

The authors wish to thank the IC process group of DIMES for technical assistance. This research is supported by the Dutch Technology Foundation, STW (project DMF .5103), Applied Science Foundation of NWO and the technology programme of Ministry of Economic Affairs.

\section{REFERENCES}

[1] M. Bao et al, Effects of electrostatic forces generated by the driving signal on capacitive sensing devices, Sensors and Actuators, A84, Issue 3, pp. 213-219.

[2] W. C. Tang, Digital Capacitive Accelero-meter, US Patent 5353641, Oct. 11, 1994.

[3] H. Yang et al. A novel operation mode for accelerometers, Pacific rim workshop on transducers and micro/nano technologies, July 22-x24, 2002, Xiamen, China, pp.303-306

[4] R. E. Ziemer, W. H. Tranter, Principles of communications systems, modulation and noise. Houghton Mifflin Company, 1985. 